\title{
Estimation of the Accuracy of Mean and Variance of Correlated Data
}

\author{
Piet M. T. Broersen
}

\begin{abstract}
Monte Carlo simulations are an important tool in computational physics or statistical mechanics. Physical constants or properties are found as the mean or the variance of successive states of simulated systems. A new method to determine the statistical accuracy of the estimated means and variances is described. It uses the parameters of an automatically selected time series model. That time series model gives an optimal description of the spectral density and of the correlation structure of correlated data which are considered as stationary or in equilibrium. The resulting accuracy estimates are close to the Cramér-Rao bound for data where the correlation is determined by a single time constant.
\end{abstract}

Index Terms - Data processing, estimation, measurement errors, modeling, signal analysis, time series.

\section{INTRODUCTION}

$\mathbf{M}$ ONTE Carlo simulations on interactions between elementary particles are often used in physics and chemistry to derive macroscopic properties from potentials or energies of successive microscopic configurations. Reaction velocity, specific heat, magnetization and similar quantities are computed from the mean and from the covariances of those energies and potentials. The aim is to extract the maximum information from the statistical data in the simulations and at the same time to estimate the accuracy of that information [1]. Estimation of the mean of successive states is a common problem in many statistical tasks. This paper adds a third alternative to the two existing classes of accuracy estimates.

The first estimate uses the theoretical expressions for the variances of sample mean and sample variance [2], [3], which contain summations of covariances of the process. Measured values for the covariance function are substituted in those expressions. The second method is obtained by dividing the data in blocks and to increase the block size until the blocks become statistically independent [1], [2]. The required number of covariances or blocks in those two methods can be detected by looking for a plateau in the variance of the mean as a function of that number. This method requires many data and looses its accuracy if the relaxation time is of the same order of magnitude as the number of observations. The third alternative is based on new developments in time series analysis: simple, efficient, and reliable parameter estimation and order selection of autoregressive (AR), moving average (MA), and combined ARMA models [4]. For estimates from a given number of observations, best orders can be selected separately for each

Manuscript received November 9, 1998.

The author is with the Department of Applied Physics, Delft University of Technology, 2600 GA Delft, The Netherlands (e-mail: broersen@ tn.tudelft.nl). Publisher Item Identifier S 0018-9456(98)09659-4. model type [5]-[7]. Afterwards, the single best AR-MA type (AR, MA, or ARMA) can be found automatically with a new statistical criterion [4]. The parameters of this final model are used to compute an approximation for the covariance function of the unknown process, which is substituted in the same expressions that are used in the first method, as described.

This paper defines the third method. It presents simulation results to compare the three methods. The third method gives always a good estimate for the accuracy; the first and second method are related and sometimes satisfactory if many data are available. However, the tightest bounds for the accuracy are found with the third method, so that method requires less data for the same accuracy.

\section{NOTATION}

The observations $x_{1}, x_{2}, \cdots, x_{N}$ are the result of $N$ consecutive measurements of a stationary stochastic process. This can be a physical process or simulations; it also might be the result of Monte Carlo or molecular dynamics simulations in computational physics, if the system is in equilibrium. The mean value of $x_{i}$ may represent the magnetization and the variance of the mean is then proportional to the susceptibility [1]. The true process has a probability distribution function that is generally unknown and that describes the joint distribution of all $x_{i}$. The theoretical expectations $E($.$) of mean, variance$ and covariance function are determined by that probability distribution function and are denoted $\mu, \sigma^{2}$, and $R(k)$, where $R(k)$ is the mathematical expectation $E\left(x_{i}-\mu\right)\left(x_{i+k}-\mu\right)$ and $\sigma^{2}$ is equal to $R(0)$. Estimators for these quantities are functions of the observations and they are given by [3]

$$
\begin{aligned}
\hat{\mu} & =\frac{1}{N} \sum_{i=1}^{N} x_{i} \\
\hat{\sigma}^{2} & =\frac{1}{N-1} \sum_{i=1}^{N}\left(x_{i}-\hat{\mu}\right)^{2} \\
\hat{R}(k) & =\frac{1}{N} \sum_{i=1}^{N-k}\left(x_{i}-\hat{\mu}\right)\left(x_{i+k}-\hat{\mu}\right), \quad k \geq 0 ; \\
\hat{R}(-k) & =\hat{R}(k) .
\end{aligned}
$$

The mathematical expectations of those estimators are [3]

$$
\begin{aligned}
E[\hat{\mu}] & =\mu \quad E\left[\hat{\sigma}^{2}\right]=\sigma^{2} \\
E[\hat{R}(k)] & =\left(1-\frac{k}{N}\right) R(k)-\left(1-\frac{k}{N}\right) \operatorname{var}[\hat{\mu}] .
\end{aligned}
$$


Also expressions have been derived for the variance of the estimated mean and variance, when estimated from $N$ observations. For mean and variance, they are [3]

$$
\begin{aligned}
\operatorname{var}[\hat{\mu}] & =\frac{1}{N} \sum_{k=-N}^{N}\left(1-\frac{k}{N}\right) R(k) \\
\operatorname{var}\left[\hat{\sigma}^{2}\right] & =\frac{2}{N} \sum_{k=-N}^{n}\left(1-\frac{k}{N}\right) R^{2}(k) .
\end{aligned}
$$

For those accuracy measures, three estimators are compared. The first and eldest one is based on measured covariances [1], [3] and is denoted $\operatorname{var}_{C}$ with $C$ of covariance; another one uses the blocking method [1], [2] with $B$ as subscript, $\operatorname{var}_{B}$, and finally a new estimator is based on developments in time series analysis [4], $\operatorname{var}_{A}$ which has the subscript $A$ of AR-MA.

\section{ESTIMATION WITH CORRELATIONS}

It is known that estimated covariances have some undesirable statistical properties [3], especially a high degree of correlation between neighboring covariances. This makes it attractive to use only a part of all $N$ estimated covariances in determining the accuracy of mean and variance. The covariance estimator for the accuracy of the mean value, based on $K$ estimated covariances, has been defined as [2]

$$
\operatorname{vâr}_{C}[\hat{\mu}]=\frac{1}{N-2 K-1+K(K+1) / N} \sum_{k=-K}^{K} \hat{R}(k)
$$

where the denominator reflects the bias in the estimated covariance in (2). Likewise, an expression for the accuracy of the variance can be derived

$$
\operatorname{vâr}_{C}\left[\hat{\sigma}^{2}\right]=\frac{2}{N-K} \sum_{k=-K}^{K} \hat{R}^{2}(k) \text {. }
$$

The bias term in (2) has been neglected in this estimator for the variance of $\hat{\sigma}^{2}$. The value of $K$ has to be chosen or selected in practice. For $N \gg K$, a possible choice has been described [2]. A plateau for the variance (4) is reached as a function of $K$, which can be recognized if $N$ is very large. It is clear that the variance estimates (4) and (5) can only be accurate if the number of observations is much longer than the distance over which the data are correlated.

\section{ESTIMATION WITH BLOCKING}

The blocking method is known from computations in statistical physics [1], [2] and its derivation has been compared to the renormalization group technique. The data $x_{1}$, $x_{2}, \cdots, x_{N}$ are transformed into half as large a data set $x_{1,1}$, $x_{1,2}, \cdots, x_{1, N / 2}$ with $x_{p, i}=\frac{1}{2}\left(x_{p-1,2 i-1}+x_{p-1,2 i}\right)$. It is evident that the average of $N$ observations $x_{i}$ (or $x_{0, i}$ in the blocking notation) and the average of $N / 2$ observations $x_{1, i}$ are exactly the same, so the average is invariant under this blocking transformation. The blocking can be repeated giving $N / 2^{2}$ new observations $x_{2, i}, N / 2^{3}$ new observations $x_{3, i}$ and so on. The variance of a block and the covariance between successive blocks become

$$
\begin{aligned}
& \operatorname{var}\left[x_{p, i}\right]=\frac{1}{2^{p}} \sum_{k=-2^{p}}^{2^{p}}\left(1-\frac{k}{2^{p}}\right) R(k) \\
& \operatorname{cov}\left[x_{p, i}, x_{p, i+1}\right] \\
& \quad=\frac{1}{\left(2^{p}\right)^{2}}\left\{\sum_{k=1}^{2^{p}} k R(k)+\sum_{k=2^{p}+1}^{2^{p+1}}\left(2^{p+1}-k\right) R(k)\right\} .
\end{aligned}
$$

The second term in this covariance can be neglected for certain $p$, because $R(k)$ will be very small for $k>2^{p}$; the first term is equal to one half of the contribution of the triangular window in the block variance of (6). A comparison with (3) shows that the block variance can approximate $N / 2^{p}$ times the variance of the mean if the contribution of the window of length $2^{p}$ may be neglected. Otherwise, the blocking estimate is biased for small $p$. That bias can be diminished by adding twice the covariance of (6) to the estimated variance.

The advantage of this blocking operation is that the consecutive blocks become less and less correlated when they grow in length. Finally, the covariance between the blocks may be negligible and the variance of the mean is estimated with $N / 2^{p}$ blocks of length $2^{p}$ divided by $N / 2^{p}-1$ [2], so

$$
\operatorname{vâr}_{B}[\hat{\mu}]=\frac{\operatorname{vâr}\left[x_{p, i}\right]}{N / 2^{p}-1}=\frac{\sum_{p=1}^{N / 2^{p}}\left(x_{p, i}-\hat{\mu}\right)^{2}}{N / 2^{p}-1} .
$$

The derivation of this estimator has been based on a positive covariance between successive blocks for all block sizes [2]. This is not completely general: with this assumption it can be proved that the variance of blocks increases to a plateau for greater block sizes. This requires many observations, because the length of each block must be longer than the correlation length of the data and many blocks are necessary to obtain good estimates for the accuracy of mean and variance.

The blocking operation can also be seen as ordering the $1 \times N$ observations in a $2 \times N / 2$ pattern and so on; the first column contains the first $2^{p}$ observations $x_{1}, x_{2}, x_{3}, \cdots$. Then, $x_{p, i}$ is the mean value of the $i$ th column of the $2^{p} \times N / 2^{p}$ representation of the data. The advantage of this ordering is that also the variance of each column can be computed, which leads to the block type of estimator

$$
\operatorname{vâr}_{B}\left[\hat{\sigma}^{2}\right]=\frac{\operatorname{vâr}\left[\hat{\sigma}_{p, i}^{2}\right]}{N / 2^{p}-1}=\frac{\sum_{p=1}^{N / 2^{p}}\left(\hat{\sigma}_{p, i}^{2}-\hat{\sigma}^{2}\right)^{2}}{N / 2^{p}-1},
$$

where $\hat{\sigma}^{2}$ itself is estimated with (1).

In practice, one also wants to know the accuracy of the estimates of the variance [2]. A theoretical argument with asymptotical validity supposes that the block estimates are unbiased estimates that consist of $N / 2^{p}$ uncorrelated contributions. This approximation can be good for larger block sizes 
where the estimators (7) and (8) reached their plateau and the correlation between blocks may be neglected. The standard deviations of the block estimators are approximately given by

$$
\frac{\operatorname{s.d}\left\{\operatorname{vâr}_{B}[\hat{\mu}]\right\}}{\operatorname{var}[\hat{\mu}]} \approx \frac{\operatorname{s.d}\left\{\operatorname{vâr}_{B}\left[\hat{\sigma}^{2}\right]\right\}}{\operatorname{var}\left[\hat{\sigma}^{2}\right]} \approx \sqrt{\frac{1}{N / 2^{p}-1}} .
$$

In simulations, the above approximations will be compared with the experimental standard deviations and also with the root mean square (RMS) error that is defined as the square root of the sum of the variance and the squared bias.

\section{Estimation with TIME SERIES}

Time series models have three different types, autoregressive or AR, moving average or MA, and combined ARMA. $\operatorname{An} \operatorname{ARMA}(P, Q)$ process can be written as [3]

$$
\begin{aligned}
& x_{n}+a_{1} x_{n-1}+\cdots+a_{P} x_{n-P} \\
& \quad=\varepsilon_{n}+b_{1} \varepsilon_{n-1}+\cdots+b_{Q} \varepsilon_{n-Q}
\end{aligned}
$$

where $\varepsilon_{n}$ is a purely random process, so a sequence of independent identically distributed stochastic variables. This process is $\mathrm{AR}$ for $Q=0$ and $\mathrm{MA}$ for $P=0$. Any stationary stochastic process with a continuous spectral density can be written as an unique $\operatorname{AR}(\infty)$ or $\mathrm{MA}(\infty)$ process [3], independent of the origin of the process, e.g., it may be the sum of a true AR process and a colored noise signal.

An estimated $\operatorname{ARMA}(p, q)$ model is given by

$$
\begin{aligned}
& x_{n}+\hat{a}_{1} x_{n-1}+\cdots+\hat{a}_{p} x_{n-p} \\
& \quad=\hat{\varepsilon}_{n}+\hat{b}_{1} \hat{\varepsilon}_{n-1}+\cdots+\hat{b}_{q} \hat{\varepsilon}_{n-q}
\end{aligned}
$$

where $p$ and $q$ may be arbitrary and $\hat{\varepsilon}_{n}$ is some colored noise signal that is not purely random in general. Parameters for different model orders are estimated by minimization of the residual variance, which is the variance of $\hat{\varepsilon}_{n}$. The theory shows that this minimum gives at the same time the maximal flatness for the spectral density of $\hat{\varepsilon}_{n}$. Afterwards, orders $p$ and $q$ are selected. Selection criteria add a penalty for each estimated parameter to the logarithm of the residual variance [8] of each estimated model and look for the minimum of the criterion. Models are AR if $q=0$ and purely MA for $p=0$. Moreover, finite order models are quite well in describing true processes with infinite orders, because the true parameters are decreasing rapidly for many processes.

New developments in time series can roughly be characterized. It has always been accepted that a time series model is the best possible description of the structure of the data in time or in frequency domain, if the true model type and the true model order are known. Unfortunately, the general way of producing stationary stochastic processes gives seldom the opportunity to know a priori or from physical considerations the true model type and model order. So model type and model order must be found from the data. Practical problems preventing the routine application of time series analysis so far were as follows.
- AR model parameters depend on the estimation method if model orders are not small in comparison with the number of observations; moreover, the selected model order depends on the maximum order. A new order selection criterion and the use of Burg's algorithm solved those problems [4], [5].

- MA estimation has many nonlinear algorithms, which cause some serious limitations. The practical problem is that estimated models of some orders may be useless due to computational problems of convergence. Also, zeros of the MA model may give noninvertible models. It was impossible to select automatically an unknown MA model order with those algorithms. However, Durbin's algorithm which uses an intermediate AR model has been improved by a better choice of that AR order and gives good results for all types of data [6].

- ARMA estimation had more problems. Most algorithms can fail in small samples or if roots of AR or MA part lie close to the unit circle. Only Durbin's second algorithm has no convergence problems, but initial conditions and the order of an intermediate AR model were open questions. A satisfactory solution has been given [7], but details still can be improved.

With the newest computer programs [4]-[7], all practical problems have been solved. Unknown data, if stationary and stochastic, can be analyzed; automatically a single time series model type and model order is selected. The quality of this selected model is, in numerous simulations, comparable with the quality that could be obtained if the true process type and order would be known in advance. In other words, not knowing the true process is no impediment for automatically finding the best model. Examples of computer algorithms that take care of reliable estimation and order selection have been described for AR [5], MA [6], and ARMA [7] estimation. The choice between those three types is described in [4]; the final single model is denoted the AR-MA model.

The covariance function and the power spectral density can be computed from the parameters. The power spectrum $\hat{h}(\omega)$ of the $\operatorname{AR}-\mathrm{MA}(p, q)$ model is given by [3]:

$$
\begin{gathered}
\hat{h}(\omega)=\frac{\sigma_{\hat{\varepsilon}}^{2}}{2 \pi}\left|1+\sum_{i=1}^{q} \hat{b}_{i} \exp (-j \omega i)\right|^{2} / \\
\left|1+\sum_{i=1}^{p} \hat{a}_{i} \exp (-j \omega i)\right|^{2} .
\end{gathered}
$$

Many methods can compute the covariance $\hat{R}_{A}(k)$ of a model, like inverse Fourier transform of the spectrum, a direct formula [3] for simple processes or a generally applicable derivation [9]. The quality of the power spectrum (12) is much better than the quality obtained with Fourier transform of the data or of the measured covariance function. As a consequence, substituting the time series estimates for the covariance in the formulas for the accuracy of mean and variance may be a good idea. This is the third estimate, for which asymptotical 
expressions have been derived [3]

$$
\begin{aligned}
\operatorname{vâr}_{A, a s s}[\hat{\mu}] & =\frac{\sigma_{\hat{\varepsilon}}^{2}}{N} \frac{\left(1+\sum_{i=1}^{q} \hat{b}_{i}\right)^{2}}{\left(1+\sum_{i=1}^{p} \hat{a}_{i}\right)^{2}} \\
& =\frac{2 \pi \hat{h}(0)}{N} \\
& =\frac{1}{N} \sum_{k=-\infty}^{\infty} \hat{R}_{A}(k) .
\end{aligned}
$$

Asymptotically, the variance of the mean is equal to the sum of all covariances divided by the number of observations. Also an asymptotical estimate for the accuracy of the variance

$$
\operatorname{vâr}_{A, a s s}\left[\hat{\sigma}^{2}\right]=\frac{4 \pi}{N} \int_{-\pi}^{\pi} \hat{h}^{2}(\omega) d \omega=\frac{2}{N} \sum_{k=-\infty}^{\infty} \hat{R}_{A}^{2}(k)
$$

has been derived [3]. If the true process is ARMA, those equations for vâr $A_{A}$ converge to (3), if the true parameter values are substituted. The asymptotical approximation means that all covariances from shift 0 to $\infty$ have been included, without triangular window. This representation is a good start for theoretical considerations with the Cramér-Rao lower bound. Another formulation includes the triangular window by using the $N$ covariances that can be computed from the selected and estimated AR-MA model

$$
\begin{gathered}
\operatorname{vâr}_{A}[\hat{\mu}]=\frac{1}{N} \sum_{k=-N}^{N}\left(1-\frac{k}{N}\right) \hat{R}_{A}(k) \\
\operatorname{vâr}_{A}\left[\hat{\sigma}^{2}\right]=\frac{2}{N} \sum_{k=-N}^{N}\left(1-\frac{k}{N}\right) \hat{R}_{A}^{2}(k) .
\end{gathered}
$$

It is possible to use $N$ terms in this summation. This is an advantage above blocking and covariance estimates that can use only a fraction of $N$. This may be important for finite $N$.

\section{CRAMÉR-RAO BOUNDS}

Means and variances of functions of stochastic variables can be approximated with Taylor expansions around the mean value of the function for large samples [3], but only if the variations are small in comparison with the mean. Often, the mean of the function, its mathematical expectation, can be approximated with sufficient accuracy by taking only the constant term in this expansion, because the terms with first derivatives give no contribution to the mean and the second derivatives are small. The variance of a function depends mainly on the first derivatives of the function and of course on the covariance matrix of the original stochastic variables. Now, consider the estimated parameters and the estimated residual variance of a time series AR-MA model as stochastic variables. Then, vâr ${ }_{A, a s s}[\hat{\mu}]$ and vâr $r_{A, \text { ass }}\left[\hat{\sigma}^{2}\right]$ are functions of those variables and Taylor expansion gives approximations for the mean and for the variance of the functions.

It is well known that for a given number of observations a lower bound exists for the accuracy of estimated parameters $\hat{\theta}$ : the Cramér-Rao bound [3]. Asymptotically, that lower bound can be obtained by the maximum likelihood estimator. Under rather mild conditions, the maximum likelihood estimator of a function of a stochastic variable, $\hat{v}(\theta)$, is equal to the function of the maximum likelihood estimate of the stochastic variable itself: $v(\hat{\theta})$. So the asymptotical covariance matrix of a function $\hat{v}(\hat{\theta})$, estimated with the maximum likelihood principle, is also the Cramér-Rao bound for the best asymptotically normal estimator of $\hat{v}(\theta)$ [10, p. 249]. The asymptotical lower bound for the accuracy of an unbiased estimate of $v(\theta)$ is given by the partial derivatives of $v$ to the parameters in $\theta$ and by the lower bound for the accuracy of the parameters themselves, that can be expressed in the Fisher information matrix $I(\theta)$. For a function $\hat{v}(\hat{\theta})$ of parameters $\theta$ that have as their Cramér-Rao bound $I(\theta)^{-1}$, this becomes ([10], [11, p. 47])

$$
\operatorname{var}[\hat{v}(\hat{\theta})] \geq\left[\frac{\partial v}{\partial \theta}\right] I(\theta)^{-1}\left[\frac{\partial v}{\partial \theta}\right]^{T} .
$$

This relation will be used to derive expressions for the lower bound of the asymptotical expressions for the variance of the variance of mean and variance.

Explicit formulae will be derived here for $\operatorname{AR}(1)$ processes only. The vector of estimated parameters $\hat{\theta}$ has 2 elements: $\hat{a}_{1}$ and $\sigma_{\hat{\varepsilon}}^{2}$. The variance of $\hat{a}_{1}$ is $\left(1-a_{1}^{2}\right) / N$. The Cramér-Rao lower bound for the variance of $\hat{\varepsilon}_{n}$ is $2 \sigma_{\varepsilon}^{2} / N$. The covariance between those estimates is zero [11]. With (13), the lower $\operatorname{AR}(1)$ bound for the variance of $\operatorname{var}_{A, a s s}[\hat{\mu}]$ becomes

$$
\begin{aligned}
\operatorname{var} & {\left[\operatorname{vâr}_{A, a s s}[\hat{\mu}]\right] } \\
& =\operatorname{var}\left[\frac{\sigma_{\hat{\varepsilon}}^{2}}{N} \frac{1}{\left(1+\hat{a}_{1}\right)^{2}}\right] \\
& \approx \frac{\sigma_{\varepsilon}^{4}}{N^{2}} \operatorname{var}\left[\frac{1}{\left(1+\hat{a}_{1}\right)^{2}}\right]+\frac{1}{N^{2}\left(1+a_{1}\right)^{4}} \operatorname{var}\left[\sigma_{\hat{\varepsilon}}^{2}\right] \\
& =\frac{\sigma_{\varepsilon}^{4}}{N^{2}}\left[\frac{2}{\left(1+a_{1}\right)^{3}}\right]^{2} \frac{1-a_{1}^{2}}{N}+\frac{1}{N^{2}\left(1+a_{1}\right)^{4}} \frac{2 \sigma_{\varepsilon}^{4}}{N} .
\end{aligned}
$$

For comparisons with other estimates, it is simpler to present the ratio of variance and squared expectation, yielding

$$
\frac{\operatorname{var}\left[\operatorname{vâr}_{A, a s s}[\hat{\mu}]\right]}{E^{2}\left[\operatorname{vâr}_{A, a s s}[\hat{\mu}]\right]}=\frac{6-2 a_{1}}{N\left(1+a_{1}\right)} \text {. }
$$

This expression can be compared with $1 /\left(N / 2^{p}-1\right)$ that has been derived for the variance of the blocking technique in (9). However, the blocking variance also depends on the value of $a_{1}$ because of the minimum required block length to obtain a small bias. With some exercise, it follows that

$$
\begin{aligned}
E\left[\operatorname{vâr}_{A, a s s}\left[\hat{\sigma}^{2}\right]\right] & =\frac{2 \sigma_{\varepsilon}^{4}}{N} \frac{1+a_{1}^{2}}{\left(1-a_{1}^{2}\right)^{3}} \\
\frac{\operatorname{var}\left[\operatorname{vâr}_{A, a s s}\left[\hat{\sigma}^{2}\right]\right]}{E^{2}\left[\operatorname{vâr}_{A, a s s}\left[\hat{\sigma}^{2}\right]\right]} & =\frac{8}{N}+\frac{16 a_{1}^{2}\left(2+a_{1}^{2}\right)^{2}}{N\left(1-a_{1}^{2}\right)\left(1+a_{1}^{2}\right)^{2}} .
\end{aligned}
$$

Similar results can be derived for higher order AR processes, for MA and for ARMA, but the calculus becomes extensive for higher orders of the process.

Finally, the correlation coefficient between two successive blocks can also be computed for this AR(1) process. By using 
only the first term in the covariance of (6) it is found that the normalized correlation between blocks is given by

$$
\frac{\operatorname{cov}\left[x_{p, i}, x_{p, i+1}\right]}{\operatorname{var}\left[x_{p}, i\right]} \approx \frac{1}{2^{p}} \frac{\sum_{k=1}^{2^{p}} k R(k)}{\sum_{k=-2^{p}}^{2^{p}} R(k)} \approx \frac{-a_{1}}{2^{p}\left(1-a_{1}^{2}\right)} .
$$

The bias in $\operatorname{vâr}_{B}[\hat{\mu}]$ is approximately twice this amount.

\section{SimUlations}

First of all, the properties of the correlation and blocking methods have been studied in an AR(1) example. Fig. 1 gives five realizations for blocking, as a function of the block size $2^{p}$, indicated by $p$. It becomes clear that results of successive blocking operations create an irregular pattern around the average of the plateau. The results resemble an example with measurements on an Ising model [2]. The variance of the mean is normalized by dividing by the asymptotical mathematical expectation of that variance, for the given number of observations, so that the true value in Fig. 1 should be 1. Fig. 2 gives the same process with much less observations, showing that it will become difficult to recognize a plateau in a single realization. That is completely impossible in Fig. 3, where an $\mathrm{AR}(1)$ example with a higher correlation has been taken. Blocking reaches some plateau as an indication where the value for the variance should be taken, but it may be difficult to recognize the plateau, because the variance behaves quite irregular in every realization. Only if the standard deviation of the measured variance is small enough at the plateau, that value of the variance of the mean can be found in such a figure. The standard deviation for the covariance estimators of the accuracy was always greater than that of blocking, if the same value is used for block size $2^{p}$ and for covariance length $K$. However, in all examples the plateau was reached for a smaller value for $K$ than for $2^{p}$. This agrees with previous results [2], where block estimates were clearly lower than the correlation estimates for the first blocking operations. This is explained by the bias in (6). Of course, the smallest value for the RMSE is reached just before the plateau, with a small contribution of the bias and the smallest variance of all points on the plateau. Together, covariances perform slightly better than blocking, but both methods are outperformed by the time series, as can be demonstrated with some tables.

The tables give the average RMS error for $\{\operatorname{vâr}(\hat{\mu}) / \operatorname{var}(\hat{\mu})\}$ and $\left\{\operatorname{var}\left(\hat{\sigma}^{2}\right) / \operatorname{var}\left(\hat{\sigma}^{2}\right)\right\}$ in simulations. The RMS error is used, the square root of variance plus squared bias. This is preferred above only variance or bias, because the best results for the blocking method and the correlation method were mostly obtained for biased estimates. CRB denotes the square root of the Cramér-Rao bound of (17), true denotes the result of the estimated AR(1) model in the first three tables, so of the true model type and model order; $\operatorname{var}_{A}, \operatorname{var}_{B}$, and $\operatorname{var}_{C}$ give the RMS error of (15) for AR-MA, (7) for blocking, and (4) for the substituted covariance method.

Table I gives $\operatorname{var}_{A}$ results that are representative of the practical accuracy when this method is applied. The numbers

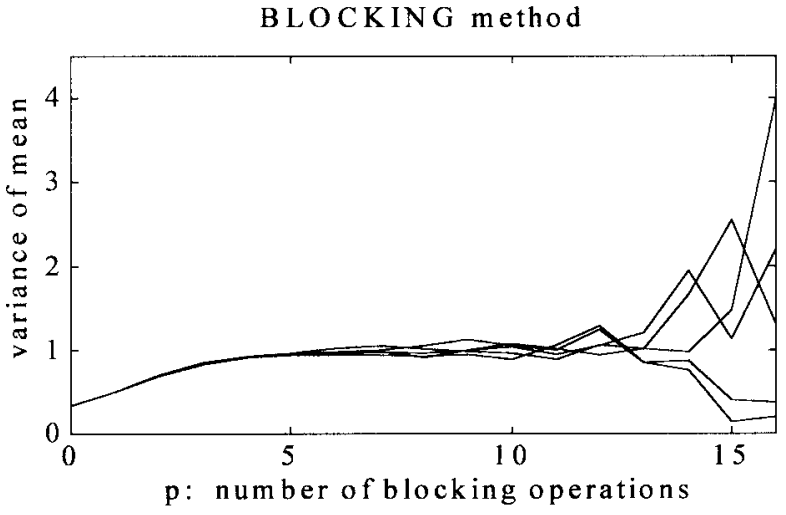

Fig. 1. Five realizations of the normalized estimated variance of the mean $\operatorname{var}(\hat{\mu}) / \operatorname{var}(\hat{\mu})$, for the blocking method with $N=2^{17}$ observations of an $\mathrm{AR}(1)$ process with $a_{1}=-0.5$, as a function of the number of blocking operations $p$, with block size $2^{p}$.

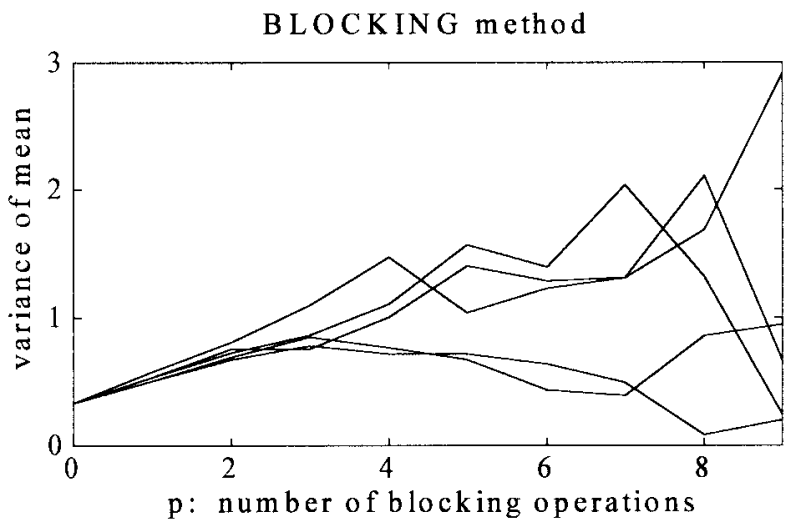

Fig. 2. Five realizations of the normalized estimated variance of the mean $\operatorname{var}(\hat{\mu}) / \operatorname{var}(\hat{\mu})$, for the blocking method with $N=2^{10}$ observations of an $\operatorname{AR}(1)$ process with $a_{1}=-0.5$, as a function of the number of blocking operations $p$, with block size $2^{p}$.

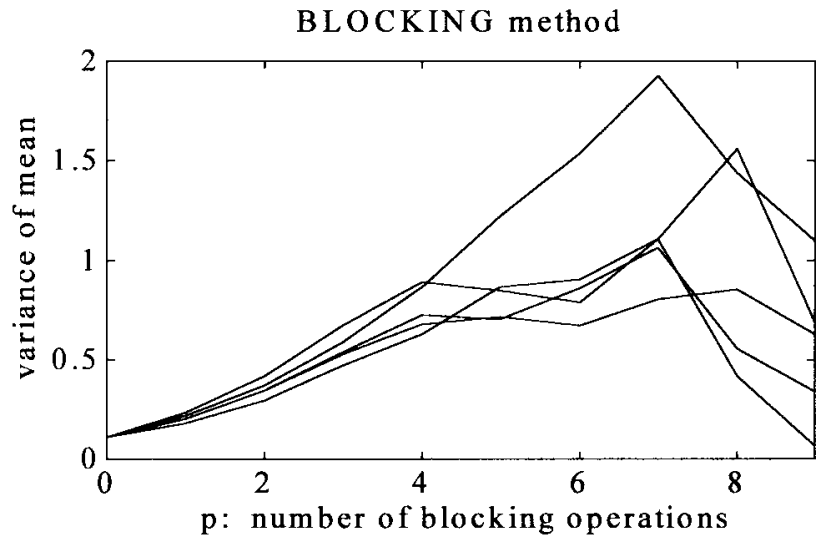

Fig. 3. Five realizations of the normalized estimated variance of the mean $\operatorname{var}(\hat{\mu}) / \operatorname{var}(\hat{\mu})$, for the blocking method with $N=2^{10}$ observations of an $\operatorname{AR}(1)$ process with $a_{1}=-0.8$, as a function of the number of blocking operations $p$, with block size $2^{p}$.

given for $\operatorname{var}_{B}$ and for $\operatorname{var}_{C}$ are not feasible in practice. The block size and covariance length with the lowest RMS value as presented here have been selected after the simulations. The best results in blocking are obtained just before the plateau is 
TABLE I

RoOt-MEAN-SQuare Error of $\operatorname{var}(\hat{\mu}) / \operatorname{var}(\hat{\mu})$ IN 100 Simulations as a Function of the Number of Observations $N$, fOR an AR(1) Process with Parameter -0.5

\begin{tabular}{c|ccccc}
\hline${ }^{2} \operatorname{logN}$ & CRB & true & $\operatorname{var}_{\mathrm{A}}$ & $\operatorname{var}_{\mathrm{B}}$ & $\operatorname{var}_{\mathrm{C}}$ \\
\hline 8 & .234 & .219 & .233 & .245 & .197 \\
10 & .117 & .109 & .122 & .166 & .126 \\
12 & .058 & .063 & .066 & .113 & .082 \\
14 & .029 & .029 & .033 & .077 & .040 \\
16 & .015 & .015 & .018 & .038 & .020 \\
\hline
\end{tabular}

TABLE II

RoOT-MEAN-SQuARE ERroR OF $\operatorname{var}(\hat{\mu}) / \operatorname{var}(\hat{\mu})$ IN 100 SimUlations AS A Function OF THE AR(1) PARAMETER FOR 1024 ObSERVATIONS

\begin{tabular}{c|ccccc}
\hline $\mathrm{a}_{1}$ & CRB & true & $\operatorname{var}_{\mathrm{A}}$ & $\operatorname{var}_{\mathrm{B}}$ & $\operatorname{var}_{\mathrm{C}}$ \\
\hline-.8 & .192 & .199 & .207 & .253 & .226 \\
-.5 & .117 & .109 & .122 & .166 & .126 \\
-.2 & .088 & .086 & .120 & .135 & .100 \\
.2 & .067 & .064 & .088 & .146 & .125 \\
.5 & .057 & .057 & .098 & .215 & .189 \\
.8 & .049 & .047 & .074 & .342 & .643 \\
\hline
\end{tabular}

reached. Some bias and a relatively small variance contribution give the smallest RMS As the single number for $\operatorname{var}_{A}$ is in almost all examples smaller than the minimum of $\operatorname{var}_{B}$ or $\operatorname{var}_{C}$ this comparison is fair. For all sample sizes, the time series AR-MA solution is close to the Cramér-Rao bound and suffers little from not knowing model type and order, as follows from a comparison with the column true. The difference between time series and blocking increases strongly for greater $N$; blocking requires over four times more observations for the same accuracy. Parameter estimates for small $N$ can be used with the Cramér-Rao theory of Section VI to compute the number of observations for obtaining some desired accuracy. The results for small $N$ can be used to extrapolate the accuracy for time series; for the blocking and covariance methods, this is not easily possible.

AR(1) processes with positive and negative parameters are treated in Tables II and III. In all examples the columns true and $\mathrm{CRB}$ are close, so the time series model of the true structure gives the best possible estimates for the accuracy. The greatest differences are found in the final row of Table II. The AR process with parameter 0.8 has the alternating correlation $1,-0.8,0.64$, and so on. This is no problem for the time series solution, but it is for the blocking and the covariance approaches. Positive AR parameters give a negative value for $R(1)$. In the blocking method, this has the consequence that the plateau is approached from above. For the covariance method, increasing $K$ gives alternating above and below the plateau, before it is reached. The positive values for $a_{1}$ have a moderate influence on $\operatorname{var}_{A}$, but a much stronger on $\operatorname{var}_{B}$ and $\operatorname{var}_{C}$.

Table III shows that all methods can give good results for the accuracy of the estimated variance. Remember that the numbers in the last two columns are too optimistic because they don't take into account the detection of the plateau. When estimating the accuracy of the variance, such a plateau also exists for the blocking method. The normalized standard deviation in (9) applies to variance estimates if the blocks
TABLE III

RoOT-MEAN-SQuare ERror of vâr $\left(\hat{\sigma}^{2}\right) / \operatorname{var}\left(\hat{\sigma}^{2}\right)$ IN 100 SimUlations as a Function of the AR(1) PARAmeter For 1024 Observations

\begin{tabular}{c|ccccc}
\hline $\mathbf{a}_{1}$ & CRB & true & $\operatorname{var}_{\mathrm{A}}$ & $\operatorname{var}_{\mathrm{B}}$ & $\operatorname{var}_{\mathrm{C}}$ \\
\hline-.8 & .282 & .294 & .295 & .463 & .277 \\
-.5 & .157 & .149 & .147 & .306 & .145 \\
-.2 & .101 & .099 & .100 & .156 & .098 \\
.2 & .101 & .096 & .097 & .269 & .098 \\
.5 & .157 & .159 & .157 & .238 & .154 \\
.8 & .282 & .256 & .328 & .270 & .237 \\
\hline
\end{tabular}

TABLE IV

Root Mean Square Error of $\operatorname{var}(\hat{\mu}) / \operatorname{var}(\hat{\mu})$ In 100 Simulations as a Function of the Number of ObSERVATIONS $N$ FOR A Gaussian Bell Shape Covariance: $R(i)=\exp \left(-0.125 i^{2}\right)$

\begin{tabular}{c|ccccccc}
\hline${ }^{2} \operatorname{logN}$ & true & $\operatorname{var}_{\mathrm{A}}$ & AR & MA & ARMA & $\operatorname{var}_{\mathrm{B}}$ & $\operatorname{var}_{\mathrm{C}}$ \\
\hline 9 & .325 & .232 & .470 & .151 & .283 & .246 & .157 \\
10 & .204 & .131 & .343 & .105 & .175 & .179 & .113 \\
11 & .153 & .091 & .262 & .082 & .140 & .145 & .108 \\
12 & .109 & .074 & .187 & .060 & .099 & .124 & .079 \\
13 & .076 & .049 & .142 & .045 & .068 & .098 & .056 \\
14 & .052 & .033 & .101 & .030 & .046 & .077 & .036 \\
15 & .036 & .021 & .066 & .021 & .033 & .056 & .026 \\
16 & .027 & .018 & .057 & .018 & .025 & .052 & .021 \\
\hline
\end{tabular}

are long enough. The covariance method, however, gives a continuously increasing level for increasing $K$ and gives no clear indication of a plateau and no good value for the number of estimated covariances that should be taken into account.

A last example gives a finite true correlation function with a MA (17) process, denoted true here. Tables IV and V present the average results of simulations where the true correlation function consisted of 17 points of a true Gaussian shape: $R(i)=\exp \left(-0.125 i^{2}\right)$. Only a couple of parameters are statistically significant when estimated, so the best estimated MA model has a lower order than the true process. AR, MA, and ARMA give results of selected models of types AR, MA, and ARMA, respectively. For this finite number of nonzero covariances, $\operatorname{var}_{A}$ and $\operatorname{var}_{C}$ are close, but the first one includes the selection of the model, and the number of covariances in $\operatorname{var}_{C}$ is chosen afterwards as the one giving the best result. $\operatorname{Var}_{B}$ was always greater, with an increasing difference for more observations. The fact that the selected AR-MA model was better than the true MA(17) model is caused by small true covariances. Table IV particularly shows that taking only AR or MA models for a time series analysis can be wrong. In this example MA models perform excellent and AR bad. For increasing $N$, the selected AR-MA model comes closer to the MA column, so selection of the model type hardly introduces an additional inaccuracy. If $N$ increases, selection of the model type becomes easier and the result of the selected type is very close to the result of the true type. In contrast, the difference with blocking becomes greater with $N$, probably because of the bias term in (6).

\section{CONCLUding REMARKS}

The new time series method AR-MA automatically selects a model type and a model order. Using the covariance structure 
TABLE V

Root Mean Square Error of $\operatorname{var}\left(\hat{\sigma}^{2}\right) / \operatorname{var}\left(\hat{\sigma}^{2}\right)$ IN 100 Simulations AS A FUNCTION OF THE NUMBER OF OBSERVATIONS $N$ FOR A Gaussian Bell Shape Covariance: $R(i)=\exp \left(-0.125 i^{2}\right)$

\begin{tabular}{c|ccccccc}
\hline${ }^{2} \log N$ & true & var $_{\mathrm{A}}$ & $\mathrm{AR}$ & \multicolumn{1}{c}{ MA } & $\mathrm{ARMA}$ & var $_{\mathrm{B}}$ & var $_{\mathrm{C}}$ \\
\hline 9 & .293 & .272 & .326 & .245 & .282 & .466 & .265 \\
10 & .181 & .174 & .200 & .166 & .177 & .342 & .177 \\
11 & .143 & .134 & .142 & .134 & .140 & .263 & .138 \\
12 & .096 & .092 & .097 & .092 & .097 & .259 & .096 \\
13 & .071 & .069 & .072 & .069 & .071 & .165 & .069 \\
14 & .046 & .046 & .046 & .046 & .046 & .136 & .046 \\
15 & .029 & .029 & .029 & .029 & .029 & .091 & .029 \\
16 & .025 & .024 & .025 & .024 & .025 & .081 & .025 \\
\hline
\end{tabular}

of that estimated and selected model gives good results in determining the accuracy of the estimated mean and variance of the data. In an example, the Cramér-Rao lower bound for the lowest estimation variance for that accuracy was reached.

The blocking method has been defined to include also the accuracy of the variance. It reaches a plateau if there is an abundance of data. Even then, the accuracy is not as good as with AR-MA; the difference increases with the number of observations. In given examples, blocking required up to ten times more data to obtain the same accuracy as ARMA.

Measured covariances could only offer a useful alternative if there would be a statistically reliable method to determine the best number of covariances that should be included in the estimation of the accuracy.

\section{REFERENCES}

[1] K. Binder, "Introduction," in Topics in Applied Physics, Volume 71, The Monte Carlo Method in Condensed Matter Physics, K. Binder, Ed., 2nd ed. Berlin, Germany: Springer, 1995.

[2] H. Flyvberg and H. G. Petersen, "Error estimates on averages of correlated data," J. Chem. Phys., vol. 91, pp. 461-466, 1989.

[3] M. B. Priestley, Spectral Analysis and Time Series. London, U.K.: Academic, 1981.

[4] P. M. T. Broersen, "Facts and fiction in spectral analysis," in Proc. IMTC'98 Conf., St. Paul, MN, 1998, vol. 2, pp. 1325-1330.

[5] _ "The $\mathrm{ABC}$ of autoregressive order selection criteria," in Preprints Sysid'97 Conf., Kitakyushu, Japan, July 1997, pp. 231-236.

[6] _ "The best order of long autoregressive models for moving average estimation," in Signal Processing VIII, Proc. Eusipco Conf., Trieste, Italy, 1996, pp. 799-802.

[7] _ "On orders of long AR models for ARMA estimation," in Proc. ECSAP'97 Conf., Prague, Czech Republic, June 24-27, 1997, pp. 83-86.

[8] P. M. T. Broersen and H. E. Wensink, "On the penalty factor for autoregressive order selection in finite samples," IEEE Trans. Signal Processing, vol. 44, pp. 748-752, 1996.

[9] P. M. T. Broersen, "The quality of models for ARMA processes," IEEE Trans. Signal Processing, vol. 46, pp. 1749-1752, 1998.

[10] S. Zacks, The Theory of Statistical Inference. New York: Wiley, 1971

[11] S. M. Kay, Modern Spectral Estimation, Theory and Application. Englewood Cliffs: Prentice-Hall, 1988.

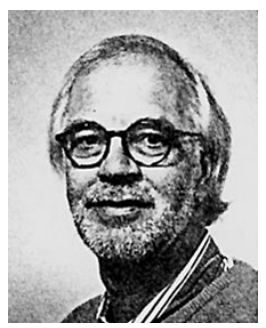

Piet M. T. Broersen was born in Zijdewind, The Netherlands, in 1944. He received the M.Sc. degree in applied physics in 1968 and the Ph.D. degree in 1976, both from the Delft University of Technology, Delft, The Netherlands.

He is currently in the Department of Applied Physics at Delft University. His research interests are the selection of order and type of time series models and the application to spectral analysis, model building, and feature extraction. 